\title{
Isotope effects of plasma chemical carbon oxidation in a magnetic field
}

\author{
Vyacheslav Fedorovich Myshkin, Valeriy Alekseevich Khan, \\ Dmitriy Aleksandrovich Izhoykin ${ }^{*}$ Ivan Alekceevich Ushakov \\ Department of Applied Physics Engineering, Institute of Physics and Technology, National Research Tomsk Polytechnic University, \\ Tomsk, Russia; 'Corresponding Author: izhoykinda@tpu.ru
}

Received 12 November 2012; revised 20 December 2012; accepted 30 December 2012

\begin{abstract}
The influence of different factors on the plasma chemical reactions is widely studied today. However, insufficient consideration is given to the research of paramagnetic phenomena which takes place in plasma systems. The results of modeling the process of redistribution carbon isotopes between different phases while oxidizing it in high-frequency low-temperature plasma in an external magnetic field are shown in the article. The equilibrium concentrations of components involved in the oxidation process in a plasma system are defined. A principle possibility of isotope-selective plasma chemical reactions in a magnetic field was experimentally determined. The increase of concentration of ${ }^{13} \mathrm{C}$ in the gas phase up to 1.3 times relative to natural abundance was obtained. It was found that the content of the carbon heavy isotope in the gas phase depends on the magnetic field action area. The best results were achieved with the combination of magnetic field impact area and the priority area of the appearance of plasma chemical reactions products.
\end{abstract}

Keywords: High-Frequency Discharge; Low-Temperature Plasma; Carbon Dioxide; Magnetic Field; Plasmachemical Reaction; Isotope Effects

\section{INTRODUCTION}

The features of plasma processes are well-known at this time [1-3]. However, insufficient attention to the paramagnetic effects in low-temperature plasma is given. These effects can significantly influence the speed ratio of various chemical processes.

The various ways to control the chemical reactions rate at room temperature are used: temperature control, reactants or products concentration change, catalysts or inhibitors usage [4]. The effect of these factors comes to change the number of particles in a reactive state. This condition is characterized by interacting atoms energy level exceeded energy barrier of chemical reaction. The energy excess of the molecule formed by a chemical reaction should be removed within a short period of time. The relaxation process of the molecule energy excess occurs through a collision with a third particle or by radiation. Although all these factors are necessary, they are insufficient. Chemical bond formation is only possible with the combination of the atomic orbitals of valence electrons having anti-parallel spine state [5].

Commonly the spins of unpaired valence electrons are oriented in a random manner. External magnetic field presence leads to ordering the spin spatial orientations of the radical's unpaired electrons and Larmor's precession around the magnetic field lines. Precession significantly changes the number of possible combinations of spins spatial orientations of the colliding atoms valence electrons. Therefore external magnetic field significantly influences on the chemical reaction conditions and the certain compounds formation. However, a similar effect is widely studied for liquid reactive systems. It is widely known that chemical reactions occurring in liquid phase in an external magnetic field are isotope-selective for magnetic and nonmagnetic nuclei [6].

${ }^{13} \mathrm{C}$ isotope enrichment due to magnetic isotope effect is established, for example, for radical reactions in the organic compound solutions [5]. This paper covers the study of an external magnetic field influence on isotope redistribution between gaseous and condensed phases of atomic carbon partial oxidation in the low-temperature plasma. Knowledge of paramagnetic phenomena mechanisms in the low-temperature plasma is necessary to development of methods of specified isotopic composition compound preparation in the gas-phase reactions.

At normal conditions the chemical reactions have insignificant isotopic selectivity due to considerable similarity of the isotopes chemical properties of an element (energy levels and reaction rates differ slightly). Therefore it is important to define processes promoting differ- 
ent isotopes fractionation to the various paths of chemical reactions. The suitable medium for the isotope selective reactions can be low temperature plasma in a magnetic field.

Low-temperature plasma is often used to increase the reactants reactivity. In low-temperature plasma conditions the bulk of substance is in the radical form. Electrons energy significantly exceeds internal energy of radicals. The electrons transfer energy to the heavy particles in the processes of atoms and molecules internal freedom degrees excitation, ionization and dissociation. Chemical active particles are created as a result of energy redistribution via these processes. Therefore major portion of energy is spent to excitation of internal freedom degrees [7].

In the low-temperature plasma particle collision time does not exceed $10^{-12}$ seconds. During this time, the spatial orientation of the valence electrons spins in a magnetic field does not change. However, in the low-temperature plasma different isotopes have the different probability to collide in a singlet state owing to different spine frequencies of the valence electron in a magnetic field [8].

The research objective is to study of the paramagnetic phenomena role in the isotope redistribution between the gaseous and condensed phases in the atomic carbon oxidation occurring in low-temperature gas-discharge plasma in an external magnetic field. The main tasks are the construction of the model describing the physical and chemical processes in low-temperature plasma in an external magnetic field and experimental research of the magnetic field influence on the isotope effects in plasma chemical reactions.

\section{MODELLING}

The process of carbon stable isotopes separation between the gaseous (carbon monoxide and carbon dioxide gases) and condensed phases (superdispersed soot) in the plasma-forming mixture of argon and carbon dioxide was studied. Atomic carbon sources are uncooled graphite electrode and carbon dioxide from the plasma-forming mixture.

Let us consider the plasma processes occurring in the magnetic field. Atomic carbon and oxygen forming the valence electrons pairs in a singlet state will vanish in each collision. Part of radicals with a random spines distribution having different thermal motion velocities will disappear in primary collision. There are equal probabilities for all available states. It is more likely that in the chemical reaction radicals having collision frequency not multiplied to frequencies of paramagnetic resonance of interacting atoms will take part in each following collision of particles having specified rate of thermal motion.
At the next collision the concentration of reagents having collision frequencies multiplied to paramagnetic resonance frequency of separate radicals will be less. The contribution of such radicals to the formation of reaction products will be small as to compare it with the rate of radical pairs forming a singlet state for the first time. Therefore the reaction products will be depleted by isotopes having spin precession frequencies multiplied radical collision frequency with the thermal motion in low-temperature plasma.

The restoration rate of spatial equilibrium distribution of radicals in the same magnetic field depends on the $g$-factor and presence of the nucleus magnetic field. Restoration of the equilibrium spatial distribution of the radical pairs via collisions under thermal motion follows the law [9]:

$$
S(t)=S(0) \exp \left(-t / \tau_{S}\right)
$$

where $\tau_{S}$-spin relaxation time.

In gases the spin-spin interaction makes the main contribution to the spin relaxation. Spin relaxation time can be defined from the electron paramagnetic resonance width:

$$
\tau_{S}=2(\gamma \Delta H)^{-1}
$$

where $\gamma-$ gyromagnetic ratio, $\Delta H$-EPR line width.

To estimate the isotope effect in the plasmachemical reactions in a magnetic field you need to model the valence electron spin dynamics with paying consideration to the equilibrium distribution restoration. Let us define the equations considering the processes occurring in lowtemperature reactive plasma placed in an external magnetic field.

Electron paramagnetic resonance (EPR) frequency is equal to unpaired electron spin precession frequency and can be defined from the expression:

$$
\omega=\beta g(H+a) / \hbar
$$

where $\beta$-Bohr magneton, $g$-gyromagnentic constant, $H$-external magnetic field value, $a$-hyperfine interaction constant, $\hbar$-Planck constant.

The collision frequency of carbon and oxygen atoms in a time unit per volume unit is:

$$
Z_{\mathrm{CO}}=n_{\mathrm{C}} n_{\mathrm{O}} d_{\mathrm{CO}}^{2} 4 \pi \sqrt{(8 \pi R T / M)}
$$

where $n_{\mathrm{C}}, n_{\mathrm{O}}$-concentration of carbon and oxygen atoms respectively, $d_{\mathrm{CO}}=0.5\left(d_{\mathrm{C}}+d_{\mathrm{O}}\right)$-the average diameter of the colliding atoms, $M=m_{\mathrm{C}} m_{\mathrm{O}} /\left(m_{\mathrm{C}}+m_{\mathrm{C}}\right)-$ reduced (given) mass of the carbon and oxygen atoms, $R$ - gas constant, $T$-absolute temperature.

The part of the colliding atoms having energy exceeded the reaction activation energy is: 


$$
\delta_{\mathrm{CO}}=\left(\frac{E_{a}}{R T}+1\right) \exp \left(-\frac{E_{a}}{R T}\right)
$$

where $E_{a}$-reaction activation energy.

Collision frequencies depend on temperature and pressure. However, during the carbon oxidation in plasma at pressure less than 1 atmosphere the plant productivity decreases due to low concentrations of the reactants.

The electron paramagnetic resonance frequencies for the carbon isotopes with atomic masses of 12 and 13 were estimated to evaluate the magnetic field influence on the carbon isotopes redistribution between phases in the high-frequency plasma torch discharge.

The Zeeman splitting of the electron energy in the magnetic field is:

$$
E=g \beta(H+a)
$$

where $\beta=9.2741 \times 10^{-24} \mathrm{~J} / \mathrm{T}, a=6.6 \mathrm{mT}$ (hyperfine splitting constant of ${ }^{13} \mathrm{C}$ for electrons on $2 \mathrm{p}$ orbital), ${ }^{12} \mathrm{~g}=$ $2.0027,{ }^{13} \mathrm{~g}=2.003$.

Electron paramagnetic resonance frequency is respectively equal:

$$
f=g \beta(H+a) / h
$$

EPR frequencies of carbon isotopes in the $0.2 \mathrm{~T}$ magnetic field are:

$$
\begin{gathered}
{ }^{12} f_{E P R}=g \beta H / h=5.5943494 \mathrm{GHz} \\
{ }^{13} f_{E P R}=g \beta(H+a) / h=5.6321156 \mathrm{GHz}
\end{gathered}
$$

Average square velocity of the atoms thermal motion can be found as:

$$
\langle v\rangle=\sqrt{8 R T / \pi M}
$$

So the velocity of ${ }^{12} \mathrm{C}$ at $2000 \mathrm{~K}$ is $1878.17 \mathrm{~m} / \mathrm{s}$, and velocity of ${ }^{13} \mathrm{C}$ is $1804.49 \mathrm{~m} / \mathrm{s}$.

Isotope path length during one period of the valence electron spine precession is:

$$
l_{E P R}=\langle v\rangle / f_{E P R} .
$$

The spines of the valence electrons of ${ }^{12} C$ and ${ }^{13} C$ make one precession turn with path 0.336 microns and 0.320 microns respectively.

The approximate expression for the dependence of the average free path from the pressure is:

$$
P=0.63 / \lambda
$$

where $\lambda[\mathrm{m}], P[\mathrm{~Pa}]$.

Thus there is one period of the valence electron spin precession of the selected isotope.

For ${ }^{12} \mathrm{C}$ the pressure discriminating the oxidation process is $18.75 \times 10^{5} \mathrm{~Pa}$. At this pressure a part of ${ }^{12} \mathrm{C}$ is oxidized at the first collision with oxygen atoms. Atoms ${ }^{12} \mathrm{C}$ which are not reacted at the first collision collide repeatedly in phase corresponding triplet state of valence electrons spines. In the following collisions a gap in the distribution of electron spin precession is formed for another polarization plane. Therefore ${ }^{12} C$ oxidation process will be less than for isotropic spin spatial orientation.

To determine the most important components of the carbon oxidation process in the plasma torch the equilibrium composition of argon + carbon + carbon dioxide in the temperature range from 100 to $3000 \mathrm{~K}$ was modeled [10]. Distribution of equilibrium concentrations is presented in Figure 1.

This temperature range corresponds to the temperature distribution at the height of the plasma torch in an experimental plasmachemical reactor of high-frequency torch plasmatron. The highest temperature is in the area of the plasma torch near the graphite electrode. In the reaction products formation area the temperature is significantly low.

Thermodynamic modeling shows that the intensive disperse phase formation is taken place in the relatively low-temperature area (less than $1500 \mathrm{~K}$ ). Almost all unoxidized carbon is in the disperse phase at temperature less than $800 \mathrm{~K}$. Maximum concentration of atomic carbon is reached in near-electrode zone.

The most significant atomic and molecular oxygen concentrations correspond to temperature range from 1500 to $3000 \mathrm{~K}$ and are dramatically reduced with the decrease of the temperature.

\section{EXPERIMENTAL}

The main idea of the experimental study is the isotope effect establishing in the atomic carbon oxidation in its excess in the low temperature gas-discharge plasma occurring in an external magnetic field relate to carbon oxidation process without a magnetic field.

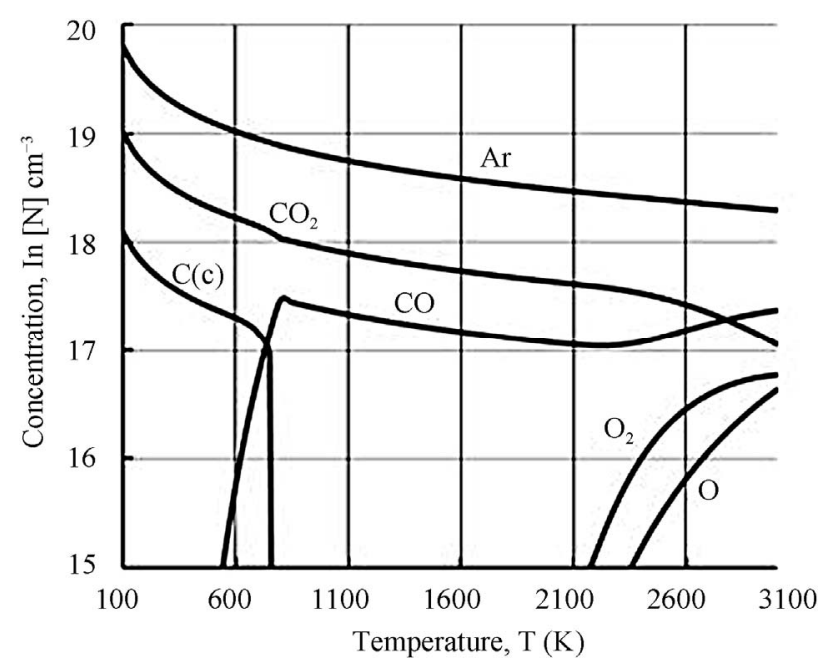

Figure 1. Equilibrium composition simulation results $(\mathrm{Ar}(84.5 \%)$ $+\mathrm{C}(0.5 \%)+\mathrm{CO}_{2}(15 \%)$, atmospheric pressure) . 
For the experimental study of magnetic field influence on chemical reactions occurring in low-temperature plasma we use the plasmachemical stand. The scheme is presented in Figure 2.

The high-frequency torch discharge is formed in quartz plasmachemical reactor using the high-frequency generator with vibrational power $4 \mathrm{~kW}$ on the frequency of 27.2 MHz. Plasma forming gas is fed tangentially into the upper part of the plasma-chemical reactor. The cold gas moves along the walls of the reactor to a high-frequency electrode. The gas heats and promotes a graphite electrode heating and evaporation. A quartz tube will cool simultaneously. The plasma flow cools in the axial region when moving along the reactor. The dispersed carbon particles are formed simultaneously.

We forced carbon atoms to go to the plasma flow in two ways. In the first case an atomic carbon was formed by graphite electrode evaporation. The average graphite evaporation rate reached up to $0.5 \mathrm{mg} / \mathrm{s}$ at plasma-forming gas flow rate $500 \mathrm{~cm}^{3} / \mathrm{s}$. In the second case atomic carbon was formed as a result of carbon dioxide thermal decomposition.

Magnetic field (0.2 $\mathrm{T}$ at the flow axis) was created using permanent magnets located outside the plasma reactor. The region of magnetic field and quenching was significantly overlapped. Magnets were placed at different distances relative to high-frequency electrodes.

The different length plasmachemical reactors for plasma flow formation were used. It helped to have different temperature at the area superposed with the magnetic field. The quenching unit geometrical parameters with external water-cooled magnets were remained constant.

The gas phase sample extraction was produced by using a submersible sampler with inner diameter $8 \mathrm{~mm}$ after graphite electrode heating and the intensive carbon evaporation. The submersible sampler was produced

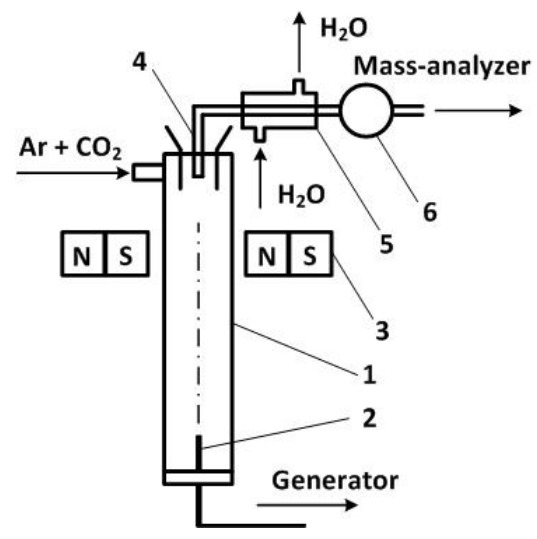

Figure 2. Experimental apparatus scheme. 1 , plasmachemical reactor, 2, high frequency electrode, 3, constant magnet, 4, submersible sampler, 5, cooling jacket, 6 , pump. from quartz glass, with output expanding nozzle in the axial region of the plasma flow. Analysis of the gas phase composition was made by mass-spectrometric complex EMG-20-7 having resolution at least 400 in the region of mass numbers 40 - 50 amu.

It was experimentally established that the increase of ${ }^{13} \mathrm{C}$ concentration in the reaction products relative to natural content at atmospheric pressure depends on the distance between the magnetic field application domain and the end of the high-voltage electrode.

It was shown that the magnetic field has better impact on the flow zone superposed with the atomic carbon oxidation area. Therefore, the influence of the external magnetic field $(0.2 \mathrm{~T})$ location that is relative to the plasma torch was studied to increase the content of the carbon heavy isotope in gaseous products of plasmachemical reactions. The experimental dependence of ${ }^{13} \mathrm{CO}_{2}$ content in carbon dioxide in the products of plasma processes on the distance between the plasma torch separation point from the electrode and the magnetic field center is shown in Figure 3.

It was established that the concentration increase of ${ }^{13} \mathrm{C}$ in the gas phase of chemical reaction products is reached by magnetic field influence on the several regions of the plasma torch. The decrease of the isotope separation effectiveness with increasing the distance from the greatest temperature area was connected with the decrease of intensity of the formation of gaseous reaction products. A significant increase of the concentration of heavy carbon isotope did not occur by enhancing the distance with the same magnetic field value due to significant temperature drop and almost complete disappearance of plasma torch atomic components.

\section{CONCLUSIONS}

The possibility of a magnetic field influence on the isotope-selective plasma processes is shown. The phenomenological model of carbon stable isotope separation between the gas and the dispersed phase in the magnetic field are proposed. The isotopic enrichment in the plasma carbon oxidation products in a magnetic field is experimentally determined.

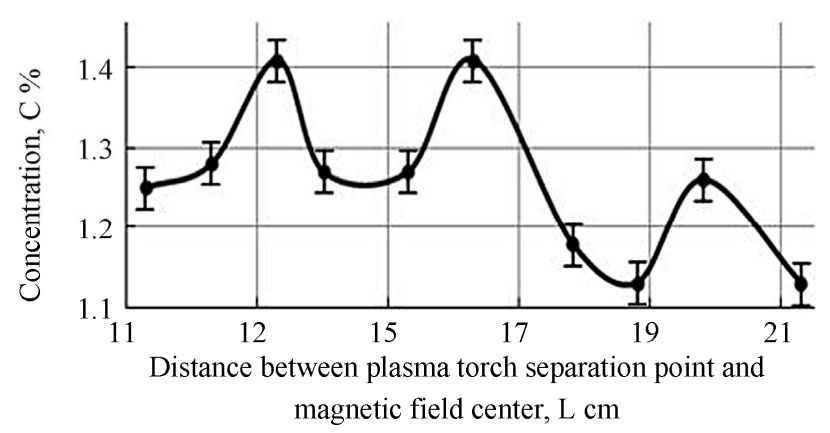

Figure 3. ${ }^{13} \mathrm{C}$ content in the carbon oxidation products $\left(\mathrm{CO}_{2}\right)$. 
The results obtained in this research can be used to study the magnetic field influence on the gas-phase chemical reactions.

\section{REFERENCES}

[1] Blaustein, B.D. (1969) Chemical reactions in electrical discharges. American Chemical Society, Washington DC. doi:10.1021/ba-1969-0080

[2] Lukes, P. and Locke, B.R. (2005) Plasmachemical oxidation processes in a hybrid gas-liquid electrical discharge reactor. Journal of Physics D: Applied Physics, 38, 40744081. doi:10.1088/0022-3727/38/22/010

[3] Benedikt, J. (2010) Plasma-chemical reactions: Low pressure acetylene plasmas. Journal of Physics D: Applied Physics, 43, 1-21. doi:10.1088/0022-3727/43/4/043001

[4] McCormick, R. (2010) Chemical kinetics: The rates and mechanisms of chemical reactions.

http://apchemistrynmsi.wikispaces.com/file/detail/12+Ki netics.pdf

[5] Gould, I.R., Turro, N.J. and Zimmt, M.B. (1984) Magnetic field and magnetic isotope effects on the products of organic reactions. Advances in Physical Organic Chemis- try, 20, 1-53. doi:10.1016/S0065-3160(08)60147-1

[6] Steiner, U.E. and Ulrich, T. (1989) Magnetic field effects in chemical kinetics and related phenomena. Chemical Reviews, 89, 51-147. doi:10.1021/cr00091a003

[7] Polak, L. (1974) Elementary chemical processes and kinetics in a non-equilibrium and quasi-equilibrium plasma. Pure and Applied Chemistry, 3, 307-342. doi:10.1351/pac197439030307

[8] Myshkin, V.F., Izhoykin, D.A., Ushakov, I.A. and Khan, V.A. (2012) Isotope effects modeling in plasma processes under magnetic field. Russian Physics Journal, 11, 348352.

[9] Myshkin, V.F., Vlasov, V.A., Izhoykin, D.A., Ushakov, I.A. and Khan, V.A. (2012) The investigation of carbon oxidation in gas-discharge plasma under external magnetic field. Proceedings of 7 th International Forum on Strategic Technology, Tomsk, 18-21 September 2012, 389393.

[10] Myshkin, V.F., Vlasov, V.A., Izhoykin, D.A., Ushakov, I.A. and Khan, V.A. (2012) The investigation of carbon oxidation process in argon plasma under magnetic field. Proceedings of 7th International Conference Plasma Physics and Plasma Technology, Minsk, 17-21 September 2012, 682-685. 